\title{
Development of Organoid-on-a-chip Platform for Preclinical Drug Screening
}

\author{
Sandra Carvalho', Diana Pinho ${ }^{1}$, Ana Vila ${ }^{1}$ \\ ${ }^{1}$ International Iberian Nanotechnology Laboratory \\ Avenida Mestre José Veiga s/n, 4715-330 Braga, Portugal \\ sandra.carvalho@inl.int; diana.pinho@inl.int; ana.vila@inl.int
}

\section{Extended Abstract}

Drug-discovery is a lengthy process causing a rapid increase in the global health care cost. In the current days, there is a growing need to implement reliable and predictable in vitro approaches into the early stages of the drug-discovery pipeline, particularly in the context of cancer research. Such effort has been focused on the development of patient-derived tumour organoids, 3D culture models that retain the structure and functions of the organs and display great potential for preclinical drug screening, prediction of patient outcomes, and guiding optimized therapy strategies at the individual level $[1,2]$. Compared to pre-existing models (tumour cell lines and patient-derived xenografts), tumour organoids can be scaled-up for high throughput testing with fewer ethical concerns. However, numerous challenges may hamper the implementation of this approach in a clinical setting, particularly due to the multifunctional and structural complex nature of organoids. Combining the ability of microfluidic platforms to precisely control fluid input and distribution with that of 3D tumour organoids models to recapitulate tumour organization and in vivo functions, the generation of an appropriate pre-clinical tumour model organoid-on-a-chip - can be envisaged, paving the way for personalized cancer medicine [3, 4].

In this work, a low cost microfluidic device suitable for the culturing, growing and maintenance of organoids on-chip was designed. The microfluidic device was manufactured by xurography [5,6] and consists in three major layers: bottom layer containing four round wells for cell seeding, middle layer displaying one inlet channel distributed in four small channels connected to each well, and top layer presenting four outlets and bonded to the previous two layers. Quality of the device was evaluated by optical microscopy. After, optimizations regarding dimensions, geometry, and flow rates were performed in order to prevent high shear flows. Finally, and as a first approach, tumour cell spheroids derived from colorectal cancer cell line SW480 were generated on-chip. Appropriate growth of SW480 spheroids and maturation on-chip was verified and similar to that obtained on commercial well plates. Culturing and maintenance of patient-derived tumour organoids on-chip and evaluation of drug activity (widely used in cancer research) on such new pre-clinical model are being performed.

Keywords: Organoid-on-a-chip, microfluidic devices, in vitro drug screening.

\section{Acknowledgements}

The project ( $\mathrm{N}^{\circ}$ Contract EAPA_384/2016) is co-financed by the European Regional Development Fund through the Interreg Atlantic Area Programme (2014-2020).

\section{References}

[1] F. Weeber, et al., "Tumor Organoids as a Pre-clinical Cancer Model for Drug Discovery," Cell Chemical Biology, vol. 24, no. 9, pp. 1092-1100, 2017.

[2] G. Kaushik, M. P. Ponnusamy, and S. K. Batra, "Concise Review: Current Status of Three-Dimensional Organoids as Preclinical Models," STEM CELLS, vol. 36, no. 9, pp. 1329-1340, 2018.

[3] M. Turetta, et al., "Emerging Technologies for Cancer Research: Towards Personalized Medicine with Microfluidic Platforms and 3D Tumor Models," Current medicinal chemistry, vol. 25, no. 35, pp. 4616-4637, 2018.

[4] F. Yu, W. Hunziker, and D. Choudhury, "Engineering Microfluidic Organoid-on-a-Chip Platforms," Micromachines, vol. 10, no. 3, 2019. 
[5] E. Pinto, et al., "A Rapid and Low-Cost Nonlithographic Method to Fabricate Biomedical Microdevices for Blood Flow Analysis," Micromachines, vol. 6, no. 1, pp. 121-135, 2015.

[6] S. P. Kojic, G. M. Stojanovic, and V. Radonic, "Novel Cost-Effective Microfluidic Chip Based on Hybrid Fabrication and Its Comprehensive Characterization," Sensors, vol. 19, no. 7, pp. 1719, 2019. 